Doping of Self-Catalyzed Nanowires under the Influence of Droplets

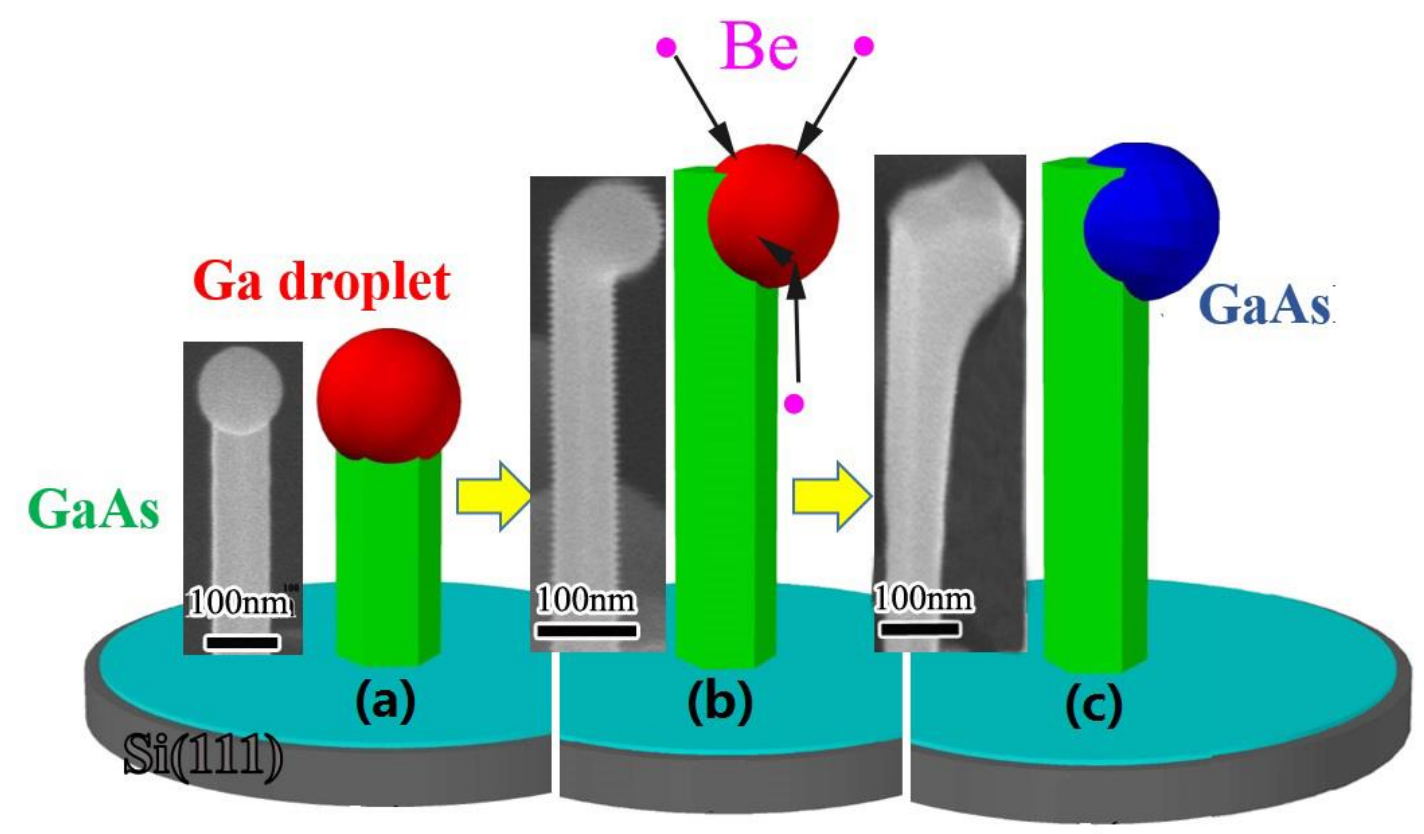




\section{Doping of Self-Catalyzed Nanowires under the Influence of Droplets}

Yunyan Zhang,,$^{\dagger^{*}}$ Zhiyuan Sun,,$^{{ }^{\perp}}$ Ana M. Sanchez, ${ }^{\perp \perp}$ Manfred Ramsteiner, ${ }^{\perp}$ Martin Aagesen, ${ }^{\|}$Jiang Wu, ${ }^{\dagger}$ Dongyoung Kim,${ }^{\dagger}$ Pamela Jurczak, ${ }^{\dagger}$ Suguo Huo, ${ }^{\mathbb{I}}$ Lincoln J. Lauhon, ${ }^{\dagger}$ and Huiyun Liu ${ }^{\dagger}$

$†$ Department of Electronic and Electrical Engineering, University College London, London WC1E 7JE, United Kingdom;

† Department of Materials Science and Engineering, Northwestern University, 2220 Campus Drive, Evanston, Illinois 60208-3108, United States

\# Department of Physics, University of Warwick, Coventry CV4 7AL, United Kingdom

$\S$ Paul-Drude-Institut für Festkörperelektronik, Hausvogteiplatz 5-7, 10117 Berlin, Germany

\| Center for Quantum Devices, Niels Bohr Institute, University of Copenhagen, Universitetsparken 5, 2100 Copenhagen, Denmark

II London Centre for Nanotechnology, University College London, London WC1H 0AH, United Kingdom

Abstract: Controlled and reproducible doping is essential for nanowires (NWs) to realize their functions. However, for the widely-used self-catalyzed vapor-liquid-solid (VLS) growth mode, the doping mechanism is far from clear, as the participation of the nanoscale liquid phase makes the doping environment highly complex and significantly different from that of the thin film growth. Here, the doping mechanism of selfcatalyzed NWs and the influence of self-catalytic droplets on the doping process are systematically studied using beryllium (Be) doped GaAs NWs. Be atoms are found for 
the first time to be incorporated into NWs predominantly through the Ga droplet that is observed to be beneficial for setting up thermodynamic equilibrium at the growth front. Be dopants are, thus substitutional on Ga sites and redundant Be atoms are accumulated inside the Ga droplets when NWs are saturated, leading to the change of the Ga droplet properties and causing the growth of phase-pure zincblende NWs. This study is an essential step toward the design and fabrication of nanowire devices.

Key words: nanowire, Ga droplet, Be doping, incorporation path, phase pure

Nanowires (NWs) have attracted significant attention, due to their novel mechanical, optical and electronic properties that are not present in the thin film counterparts $^{1-5}$ and diverse types of NW-based devices have been demonstrated across a wide range of areas, such as electronics, photonics, biochemistry and medicine ${ }^{6-13}$ Continuing improvements in individual device performance, in combination with the ability to monolithically integrate NWs onto low-cost substrates, e.g. silicon $(\mathrm{Si})$, makes them very promising for future technologies. In order to realize foundational device structures like p-i-n diodes and tunnel junctions, controlled and reproducible impurity doping is essential. Moreover, NW devices have higher requirements on doping than their bulk counter parts, such as the density and the spatial distribution of dopants. ${ }^{14}$ Understanding NW doping mechanisms is therefore a necessary prerequisite to control the NW conductivity for device fabrication.

One of the most widely used approaches to fabricate III-V NWs is vapor-liquidsolid (VLS) growth, ${ }^{15}$ also referred to as droplet-catalyzed growth, which utilizes a 
nano-sized liquid droplet to transport growth material to the liquid solid growth interface. All the materials incorporated into the core NWs need to go through them. The liquid droplet strongly influences crystal structure, ${ }^{16}$ morphology, ${ }^{17}$ and dopant incorporation ${ }^{18}$ through its chemical and mechanical interactions at the vapor-liquidsolid and liquid-solid interfaces. Due to the participation of the liquid phase, the NW growth mechanism is much more complex than those of traditional thin film structures that use the vapor-solid growth mode. Although the droplet in VLS growth effectively promotes growth along a particular direction, parasitic vapor solid (VS) growth may also occur on the exposed sidewalls of the nanowire. VLS and VS dopant incorporation pathways may produce distinct active dopant concentrations; because both pathways are influenced by growth conditions, nanowires can exhibit complex and distinct active dopant profiles. ${ }^{19}$ The complexity in growth mode brings significant challenges in understanding some fundamental principles of doping, e.g. the dominant doping path of III-V NWs. ${ }^{20,21}$

Although there have been intensive studies focusing on the mechanisms, limitations and influences of dopant incorporation on VLS-grown NW in the past few years, ${ }^{22,25-32}$ most of the reported work was conducted using foreign-metal-catalyzed growth (e.g. Au). More recently, the self-catalyzed growth mode has received increasing attention because it can avoid the possible contamination by foreign metals and has the advantage to consume the droplet for a subsequent VS shell growth. ${ }^{33}$ However, its growth condition is typically different from that of the Au-catalyzed mode. ${ }^{26,29,34,35}$ For example, self-catalyzed growth is typically conducted close to the 
melting point of the material being grown, which is often far above the growth temperature of Au-catalyzed mode. ${ }^{36,37}$ This temperature difference could influence the droplet performance and the material deposition. Thus, not all findings from foreign-metal-catalyzed growth can be transferred directly to self-catalyzed nanowires. The mechanism of doping self-catalyzed NWs is still far from clear and there still lack of consistent conclusions regarding some fundamental questions, such as the influence of the droplet in the doping process.

In this paper, the doping of self-catalyed NWs is investigated systematically using GaAs NWs and Be dopants. Be dopants are found, for the first time, to incorporate predominantly through the Ga droplet in our growth condition, which is different from previous reports that Be atoms incorporate preferentially via the NW side facets. ${ }^{27}$ The thermodynamic equilibrium maintained by the Ga droplet leads to substitutional doping of $\mathrm{Be}$ at $\mathrm{Ga}$ sites while excess Be accumulates in the Ga droplet. We also show that the formation of a Ga-Be alloy droplet alloy leads to the growth of pure zinc blende (ZB) phase NWs up to the top most bilayer. 

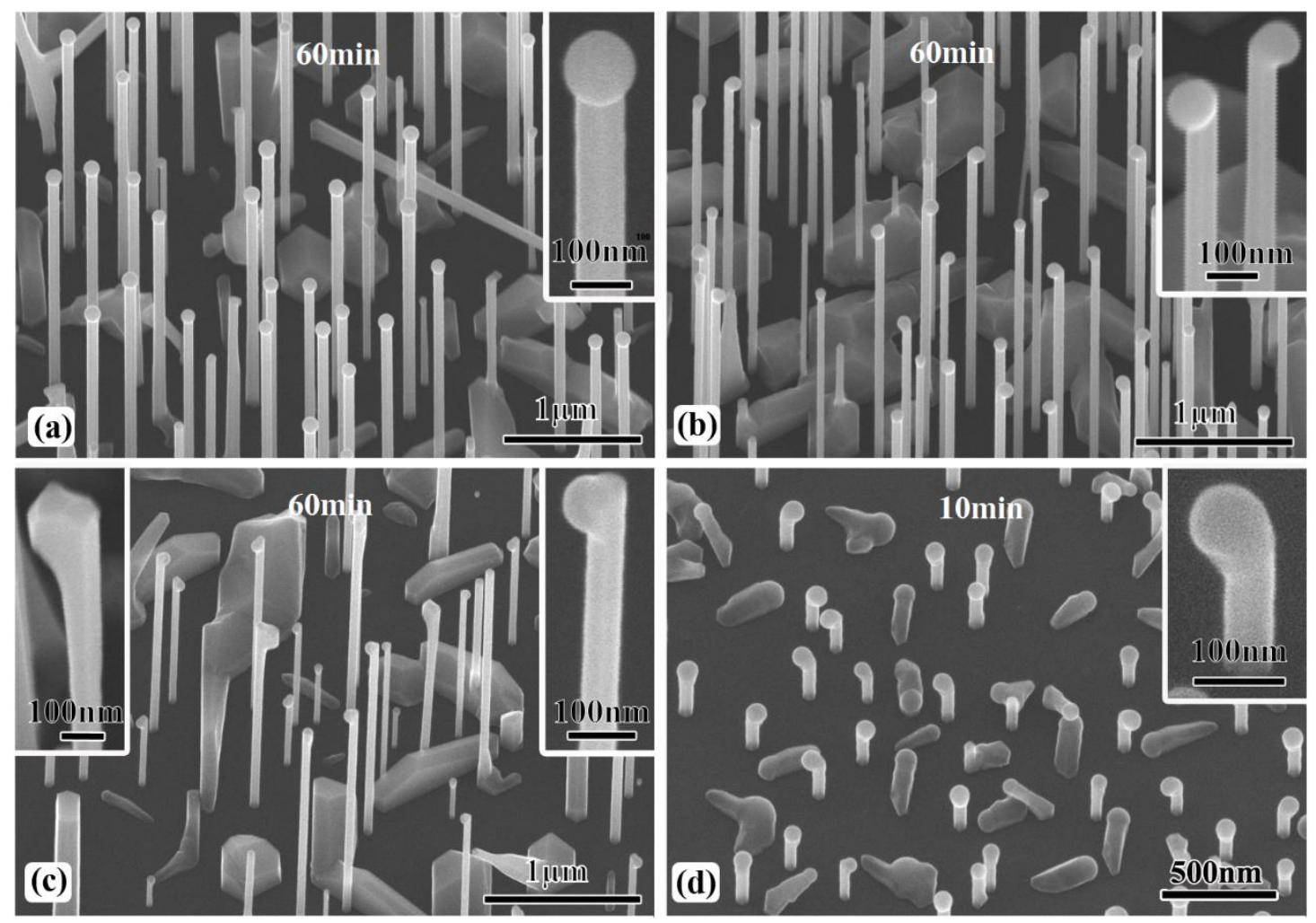

Figure 1. SEM image of GaAs NWs with nominal Be doping concentration of (a) 0, (b) $1.6 \times 10^{18}$, and (c), (d) $1.28 \times 10^{19} / \mathrm{cm}^{3}$. (a) (c) have a growth duration of 1hour, while (d) has a growth duration of only 10 minutes.

Figure 1 examines the changes in Ga droplet and GaAs nanowire morphology induced by a Be flux in a self-catalyzed growth process. During the cooling process after NW growth, both Ga and As fluxes are switched off to keep the Ga droplets. Nanowires grown without $\mathrm{Be}$ are uniform in diameter along the nanowire length (Figure 1a), indicating negligible parasitic sidewall growth, and the round Ga droplet centered at the top of each nanowire indicates a VLS growth mode. If a Be flux is introduced at a nominal concentration of $1.6 \times 10^{18} / \mathrm{cm}^{3}$, the nanowires remain uniform in diameter for a 60-minute growth, but the droplets are displaced from the nanowire center (Figure 1b), indicating that the Be changes the vapor-liquid and liquid-solid 
interface energies leading to droplet sidewall wetting. If instead growth is allowed to continue from this point for another 20 minutes, but in the absence of Be flux, a centered droplet is observed and no kinking results (Figure S1), confirming that Be induces the droplet instability. Furthermore, if the Be flux is increased to a nominal doping concentration to $1.28 \times 10^{19} / \mathrm{cm}^{3}$ (Figure 1c), the droplet slides completely to the side wall (right inset) and some of the droplets solidify (left inset) which is similar to changes in droplet morphology shown previously. ${ }^{29,38-42}$ We observe that the nanowires with intact droplets at the tip (right inset of Figure 1c, Figure 61 and Figure 6m) are uniform in diameter along their lengths, which suggests that the droplet size does not change substantially during growth. Hence, we rule out the possibility that sidewall wetting occurs due a simple increase in droplet size. Instead, the droplet instability is attributed to a surface energy imbalance caused by the increase in Be concentration inside the droplets (without a significant change in volume); this modifies the surface energies to the point that the maximum stable contact angle is exceeded. ${ }^{43}$

To test this hypothesis, nanowires were grown for a shorter duration of 10 mins at the same Be flux. As can be seen in Figure 1d, the nanowires are uniform in diameter along their lengths. Furthermore, the nanowires are terminated with a droplet at the top, and none of the droplets are on the sidewalls, consistent with lower Be concentration inside the droplets compared to the sample shown in Figure 1c. Indeed, the nanowires in Figure 1d are a case intermediate to that of Figure $1 \mathrm{~b}$ and $\mathrm{c}$. Hence, we propose that the Be concentration increases gradually during growth. At some critical concentration, the droplet wets the sidewall and then slides off the nanowire top, leading to upward 


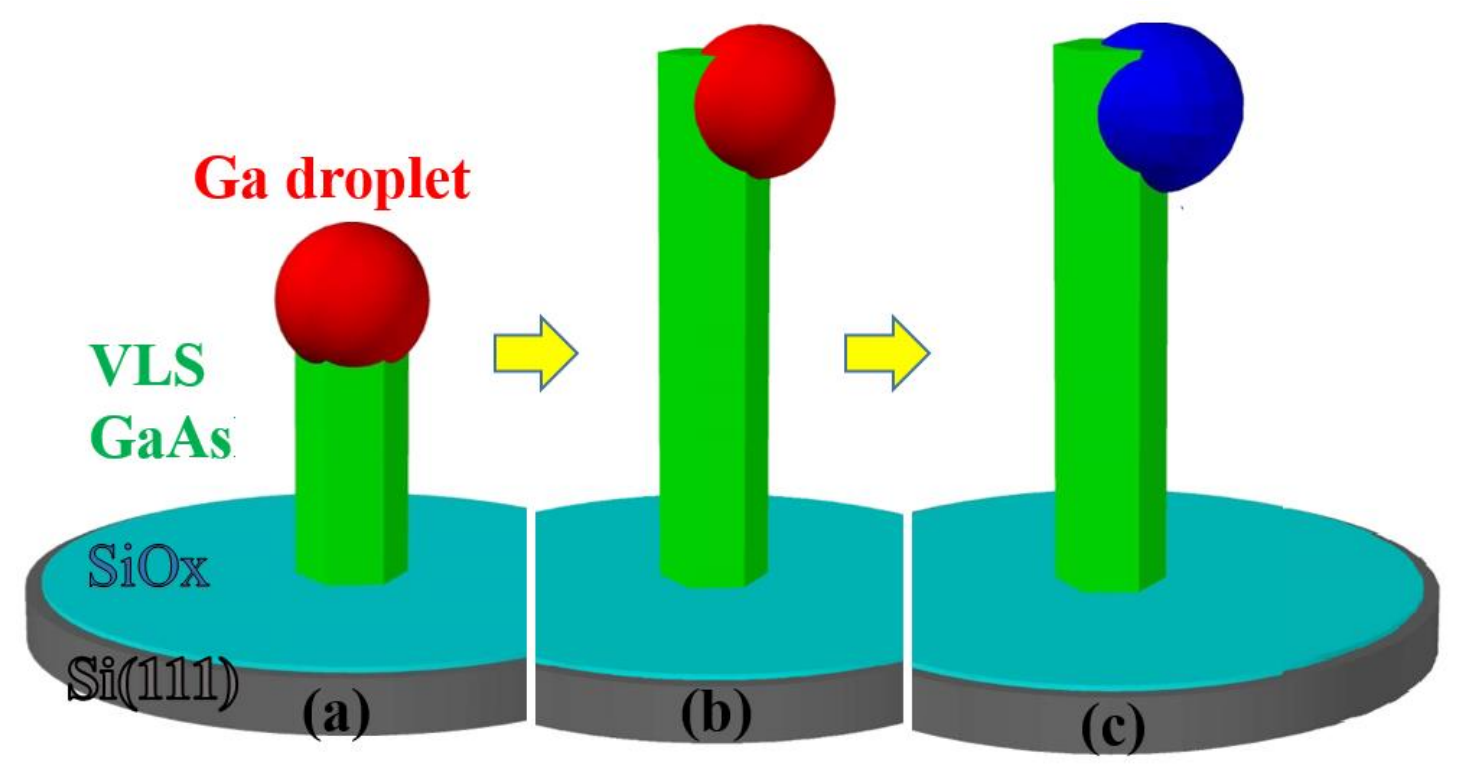

Figure 2. Illustration of growth mechanism in the self-catalyzed NW growth with Be doping. (a) Initial VLS NW growth. (b) Sidewall wetting of the droplet due to the Be accumulation and hence change of surface energy. (c) Solidification of Ga droplet.

The VLS-growth failure induced by Be doping can thus be explained by the $\mathrm{Be}$ accumulation model shown in Figure 2. At an early stage shown in Figure 2a, the NW is growing normally similarly to undoped NWs with a droplet on top. Because the NW sidewalls consist of $\{110\}$ facets that with low surface energy, parasitic VS growth at this high growth temperature is limited, and thus the NWs have a uniform diameter. ${ }^{[44,45]}$ Not all the Be can be incorporated into the NW crystal and instead accumulates inside the Ga droplet. Thus, the Be concentration increases gradually during VLS growth until the change in Ga droplet surface energy causes sidewall wetting and the loss of the droplet from the top of the NW (Figure 2b). The droplet subsequently solidifies on the sidewalls and new facets are created (Figure 2c). 

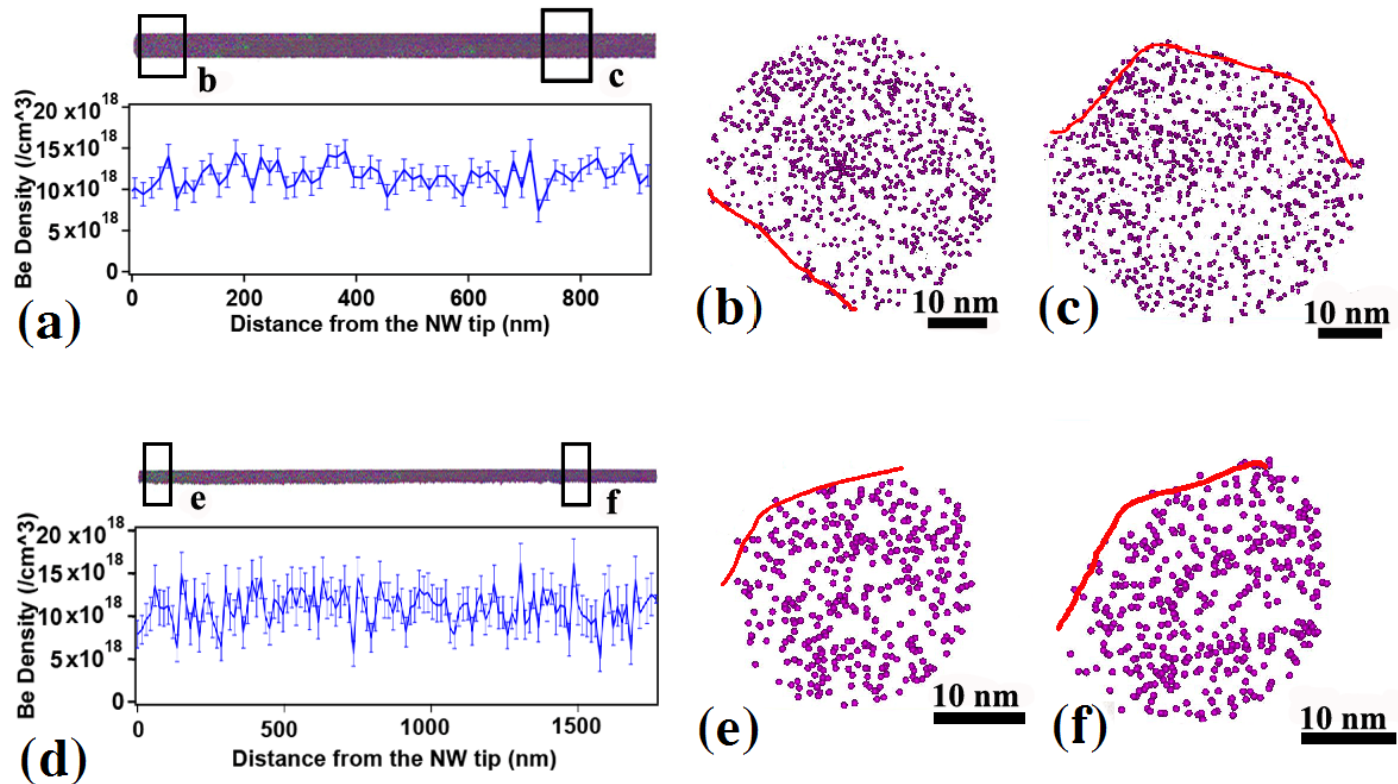

Figure 3. APT analysis of NWs with doping density of (a) (c) $1.28 \times 10^{19} / \mathrm{cm}^{3}$ and (d) (f) $6.4 \times 10^{18} / \mathrm{cm}^{3}$. (a) and (d) Be concentration profile along each NW length. Insets above are three-dimensional tomographic reconstructions of each NW. (b) (c) and (e) (f) Radial Be atom distribution maps of the $100 \mathrm{~nm}$ segments marked in the square of (a) and (d), respectively. Due to small measurement window, only part of NW surface (marked with red lines) can be reconstructed.

The invariant diameter of the nanowires indicates that VLS growth dominates sidewall (vapor-solid) growth, so a VLS dopant incorporation mechanism might also be expected. However, the accumulation of $\mathrm{Be}$ in the droplet implies that the dopant incorporation rate across the liquid-solid interface may not be simply proportional to the Be flux. Furthermore, prior studies have made conflicting claims regarding the dopant incorporation mechanisms, albeit using growth conditions that vary in the growth temperatures and III-V flux ratios. ${ }^{26}$ To clarify the dominant incorporation 
mechanism for Ga-assisted VLS growth of GaAs nanowires, atom-probe tomography (APT) measurements ${ }^{46-48}$ were performed to 60 -min-grown NWs with nominal doping concentrations of $1.28 \times 10^{19} / \mathrm{cm}^{3}$ (Sample A) and $6.4 \times 10^{18} / \mathrm{cm}^{3}$ (Sample B). The NWs used in the measurement are all uniform in diameter along the length, showing minute parasitic sidewall growth. Sample B has a length of $\sim 3.6 \mu \mathrm{m}$. In contrast, Sample A has a length of $\sim 2 \mu \mathrm{m}$ and its droplet is on the sidewall (Figure 51 and $\mathrm{m}$ ), indicating that the axial growth stopped earlier than that of Sample B and/or the dissolved Be reduced the As flux at the liquid-solid interface. ${ }^{49}$ The APT measurement started from the nanowire tip and encompasses more than half of the total length. We find that nanowires A and $\mathrm{B}$ are uniformly doped to $\sim 1.1 \times 10^{19} / \mathrm{cm}^{3}$ both along the length of the nanowires (Figure 3a,d), and radially across the diameter (Figure 3,b,c,e,f). This core doping concentration is comparable to that found by Boulanger et al, ${ }^{50}$ who used secondary ion mass spectroscopy to measure an averaged value of $\sim 5 \times 10^{18} / \mathrm{cm}^{3}$, which corresponded to the nominal doping level based on the Be flux.

The APT analysis also allows us to directly address the dopant incorporation mechanism; tilting the nanowire with respect to the APT analysis direction, the oxidized side facets (red lines in Figure 3b,c,e,f) can be brought into the field of view (see also Figure S2). ${ }^{51}$ There is no evidence of Be accumulation at the nanowire surface or Be segregation inside the nanowire crystal, suggesting that dopant incorporation occurs predominantly through the Ga droplet. This is in contrast the conclusion of Casadei et al, ${ }^{26}$ who analyzed electrical properties at various positions along nanowires to conclude that $\mathrm{Be}$ atoms incorporate preferentially via the nanowire side facets at the 
same growth temperature, but with slightly higher V/III ratio and dopant flux. If most of the Be were diffusing in from the sidewalls of our nanowires, one would expect the tip regions (Figure 3b,e) to be less doped than the middle regions (Figure 3c,f), which is not the case.

We note that sample A was grown at twice the Be flux as sample B, yet both samples have the same dopant concentration over the measured lengths, which indicates that the steady-state rate of $\mathrm{Be}$ incorporation is limited by the solubility of $\mathrm{Be}$ in $\mathrm{GaAs}$ for the growth conditions used (and further supports doping from the liquid-solid interface). This is confirmed by the observation that the Be concentration in the $\mathrm{Ga}$ droplet increases with increasing growth time. Furthermore, Be is rejected from the growth interface of sample A at a higher rate than for sample B, which is confirmed by the observations that the droplet is destabilized earlier in growth for sample A (Figure 1c,d) compared to sample B (Figure 1b). If one assumes that the growth interface is near equilibrium, one expects the Be atoms to occupy primarily the configuration of lowest energy in the GaAs lattice. ${ }^{52}$ 


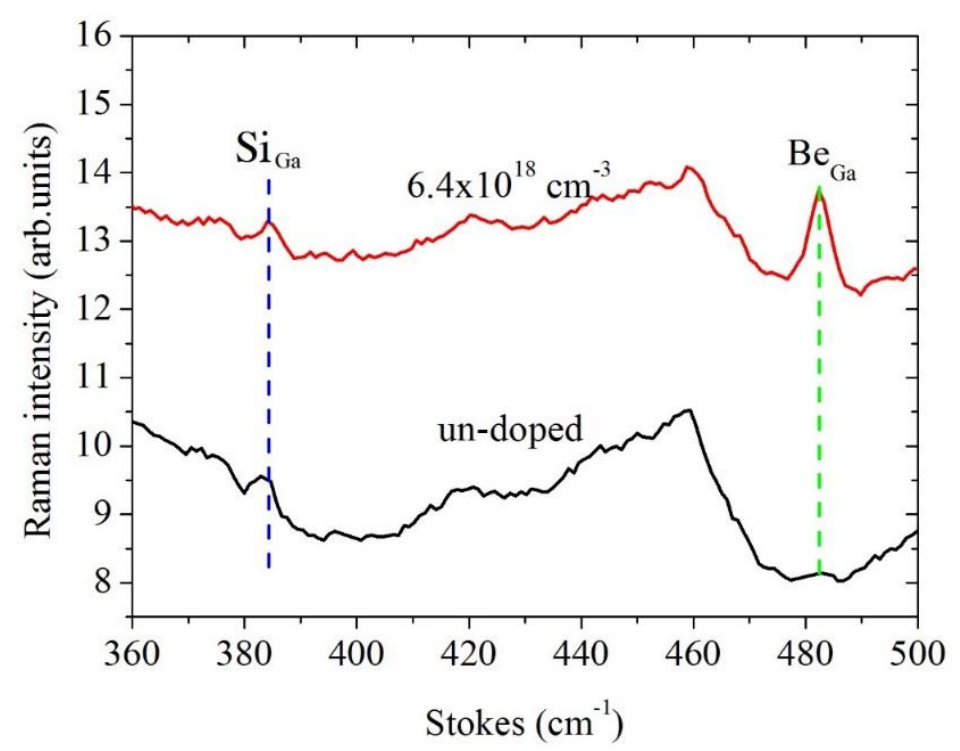

Figure 4. Raman spectra of NWs without and with doping concentration of $6.4 \times 10^{18} / \mathrm{cm}^{3}$

The configuration of Be atoms inside the Be-saturated NW crystals was studied by Raman spectroscopy of local vibrational modes (LVM). ${ }^{29}$ The Raman spectrum, shown in Figure 4, exhibits the LVM of substitutional $\mathrm{Be}$ on $\mathrm{Ga}$ site $\left(\mathrm{Be}_{\mathrm{Ga}}\right)$ at $482 \mathrm{~cm}^{-1}$ superimposed on a background of second-order phonon scattering. ${ }^{53}$ Consistent with our assignment, the relative LVM line intensity in the Raman spectrum of the Be doped nanowires corresponds to a $\mathrm{Be}_{\mathrm{Ga}}$ concentration on the order of 0.5 to $1.0 \times 10^{19} / \mathrm{cm}^{3}, 53$ which is comparable to the total concentration of incorporated Be measured by APT as shown in Figure 2.

The weaker Raman peak at $384 \mathrm{~cm}^{-1}$ has been attributed both to the LVM of Si on Ga site $\left(\mathrm{Si}_{\mathrm{Ga}}\right)^{54}$ or to Be related defects ${ }^{29}$ that were tentatively assigned to complexes including a $\mathrm{Be}$ atom on interstitial site $\left(\mathrm{Be}_{\mathrm{i}}\right)$ in GaAs NWs with extremely heavy Be doping. In order to resolve the origin of this peak in our sample, the Raman spectrum 
of nominally undoped nanowires grown under the same conditions as the doped samples is also shown in Figure 4 (black line). Since the line at $384 \mathrm{~cm}^{-1}$ is detected with comparable Raman intensity in both Be doped and nominally undoped NWs, we attribute it to $\mathrm{Si}_{\mathrm{Ga}}$ incorporated into the GaAs nanowire via the substrate, a small portion of which is dissolved by the Ga droplets. ${ }^{55}$ Therefore, these doped NWs contain predominantly $\mathrm{Be}$ atoms on Ga sites, which is consistent with the claim that the near equilibrium liquid-solid growth interface inhibits the formation of high configuration energy defect complexes. 


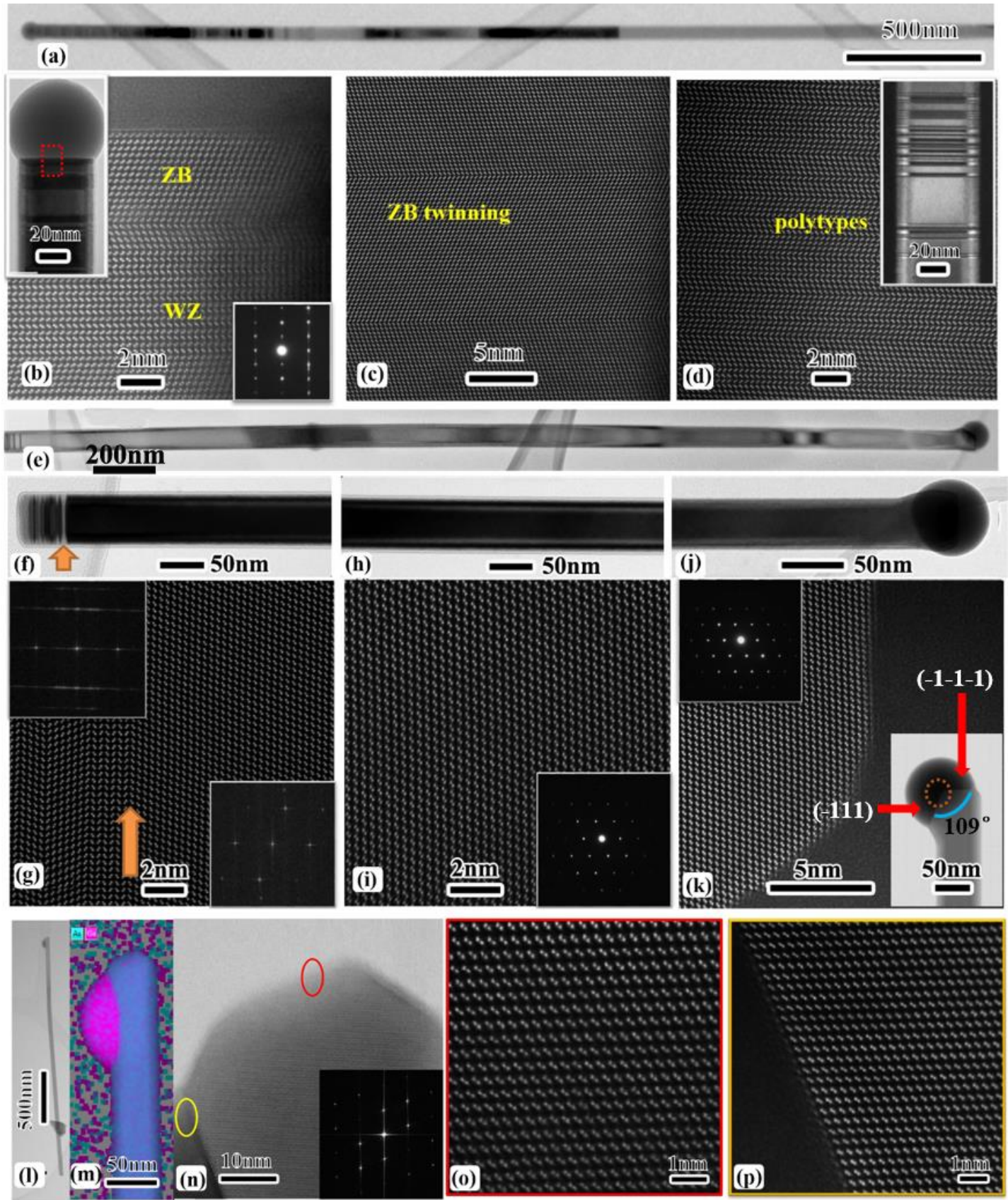

Figure 5. (a) (d) GaAs NWs without doping. (a) Low magnification TEM image of the whole NW and atomic resolution ADF-STEM image of (b) tip, (c) middle of body, and (d) bottom of the NW shown in (a). The insets in (b) are the low magnification TEM image and the selected area electron diffraction pattern of the tip portion. The insets in (d) correspond to the low magnification TEM image of the bottom portion of the NW. (e) (k) GaAs NWs with a nominal doping concentration of $1.6 \times 10^{18} / \mathrm{cm}^{3}$. (e) 
Low magnification TEM image shows the entire NW. Low magnification and atomic resolution ADF-STEM images of (f) (g) bottom, (h) (i) middle of body, and (j) (k) top of the NW. The orange arrows shown in (f) and (g) indicate similar positions. The upper left and lower right insets show the electron diffraction patterns of the defect and defectfree regions at left and right-hand side of the orange arrow, respectively. The insets in (i) corresponds to the electron diffraction pattern of (h). The insets in (k) are the low magnification TEM image and the electron diffraction pattern of the top part of the NW. (1) (p) GaAs NWs with a nominal doping concentration of $1.28 \times 10^{19} / \mathrm{cm}^{3}$. (l) Low magnification TEM image. (m) EDX mapping of the top part of the NW shown in (1). (n) Atomic resolution ADF-STEM image of the top. The inset in (n) is the electron diffraction pattern of the top part. (o) and (p) atomic resolution ADF STEM image of the are marked in (n) with a red and yellow circle respectively.

The accumulation of $\mathrm{Be}$ in the catalyst droplet has a beneficial effect on crystal structure. A typical nanowire grown without intentional doping exhibits stacking faults and polytypism as shown in TEM and Annular Dark Field (ADF)-STEM images in Figure 5a-d. The tip of the nanowire is dominated by single twins and segments of WZ and ZB (Figure 5b), the body of the nanowire contains many single twins (Figure 5c), and the bottom of the nanowire is a mixture of $\mathrm{ZB}$ and $\mathrm{WZ}$ polytypes (Figure $5 \mathrm{~d}$ ). In contrast, the nanowire grown with a Be flux has a greatly reduced defect density (Figure 5e-j). When doped with a nominal density of $1.6 \times 10^{18} / \mathrm{cm}^{3}$, the nanowire exhibits no defect-induced contrast (Figure 5e), except for a short $(\sim 50 \mathrm{~nm})$ defective segment at 
the very bottom (Figure 5f,g). From the high-resolution images shown in Figures $4 \mathrm{i}$ and $\mathrm{k}$, it is clear that above the bottom segment, the nanowire has a defect-free ZB structure up to the top-most bilayer. The suppression of WZ nucleation during growth is likely due to the lowering of droplet supersaturation and/or surface energy due $\mathrm{Be}$ accumulation to form a Be-Ga alloy droplet. ${ }^{56-58}$ It has been reported that doping can lower the droplet surface energy and increase the contact angle of the particle-NW interface, leading to an increase of the barrier for WZ nucleation. ${ }^{56}$ Consistent with this assumption, the defective region at the bottom was formed at the earliest stage of the growth, when the Be concentration inside the droplet is very low. With the increase in growth time and accumulation of Be inside the droplet, ZB nucleation becomes favored. Eventually, Be accumulation also leads to wetting of the sidewall and the development of multiple (111) growth facets. Figure 5k shows that a new (-111) facet has formed with an angle of $109^{\circ}$ relative to the (-1-1-1) facet. This is in contrast to the undoped nanowires that exhibit a single dominant (111) facet at the droplet/nanowire interface (inset of Figure 5b). For nanowires grown with a higher nominal doping density of $1.28 \times 10^{19} / \mathrm{cm}^{3}$, the droplet may move entirely to the sidewall, as seen in Figures 51 and m. The nanowire has a pure ZB crystal structure up to top most bilayer (Figure 5n-p), as observed for the nanowire in Figure 4e, and the droplet/nanowire interface also has a pure ZB crystal structure all the way to the growth front (Figure 5n,p).

In summary, Be atoms are found to incorporate into nanowires predominantly through the Ga catalyst droplets during the Ga-assisted VLS growth of GaAs nanowires by MBE. At sufficiently high Be fluxes, the Be incorporation rate saturates and 
significant amounts of $\mathrm{Be}$ are retained in the droplet. Raman analysis of local vibrational modes suggest that most Be incorporates substitutionally for Ga. This is in stark contrast to thin film vapor-solid growth at high dopant fluxes, which can result in segregation and precipitation, leading to a deterioration in material quality. ${ }^{[59-63]}$ The formation of a Be-Ga alloy droplet also has the beneficial effect of suppressing WZ nucleation and enabling the growth of a pure ZB crystal up to the top-most bilayer. These results not only guide the control and development of NW doping methods, but also provide valuable information for understanding the basic physics of doping nanoscale materials through a liquid phase.

\section{Experimental Section}

$N W$ growth: The self-catalyzed GaAs NWs were grown directly on p-type $\mathrm{Si}(111)$ substrates by means of solid-source III-V molecular beam epitaxy (MBE). ${ }^{[64,65]} \mathrm{GaAs}$ NWs were grown with a Ga beam equivalent pressure, V/III flux ratio, substrate temperature, growth duration and nominal doping concentration (characterized in thin film growth) of $8.41 \times 10^{-8}$ Torr, $44, \sim 630^{\circ} \mathrm{C}, 1$ hour and $0 \sim 1.28 \times 10^{19} / \mathrm{cm}^{3}$, respectively. The substrate temperature was measured by pyrometer.

Focus ion beam/scanning electron microscope (FIB/SEM): The NW morphology was measured with a Zeiss XB 1540 FIB/SEM system.

Transmission electron microscopy (TEM): Simple scraping of the NWs onto a lacey carbon support was used to prepare TEM specimens. The TEM measurements were performed on JEOL 2100 and doubly-corrected ARM200F microscopes, both 
operating at $200 \mathrm{kV}$.

Atom-probe tomography measurements (APT): APT samples were prepared in a Helios dual beam focused ion beam system with Kleindieck nanomanipulator. ${ }^{66}$ The NWs vertically grown on substrate were firstly welded to a sharp tungsten needle and then transferred to a silicon micropost. APT was performed on a local-electrode atomprobe tomography $4000 \mathrm{X} \mathrm{Si}$ at a base temperature of $30 \mathrm{~K}$ and base pressure of $2 \times 10^{-}$

11 Torr. The evaporation of the atoms was controlled by $355 \mathrm{~nm}$ picosecond focused laser beam at a pulse frequency of $500 \mathrm{kHz}$ and at an evaporation rate of $1 \sim 2$ atoms per 100 laser pulse. The NWs were tilted respect to the APT analysis direction intentionally during the sample preparation to enhance the APT field of view and get the surface doping information of the NW. APT data were reconstructed in the commercial software IVAS 3.6.12 (Cameca, Madison, WI). The reconstruction parameters were adjusted to reproduce the GaAs (111) interplanar distance near the 111 evaporation pole.

Raman spectroscopy: The Raman spectroscopic measurements were performed at low temperature $(10 \mathrm{~K})$ in backscattering geometry from the side facets of NWs dispersed on a gold-coated Si substrate. For optical excitation, the 405-nm line of a solid-state laser was focused by a microscope objective onto the NWs. The backscattered light was collected by the same objective, spectrally dispersed by an 80 cm spectrograph (LabRam HR Evolution, Horiba/Jobin Yvon) and detected with a liquid-nitrogen-cooled charge-coupled device. The concentration of $\mathrm{Be}$ on $\mathrm{Ga}$ sites has been estimated by the procedure described in Ref. 53. In this article, it is shown that the Be LVM Raman intensity is proportional to the concentration of Be on Ga sites. For a 
quantitative calibration, the LVM signal has been normalized to the strength of an intrinsic second-order phonon feature in the Raman spectrum. This procedure eliminates experimental difficulties in measuring absolute scattering intensities. In the case of NWs, the not exactly defined Raman scattering geometry is possibly a nonnegligible source of uncertainty in the deduced Be concentrations. Consequently, we mention in our manuscript only a relatively large concentration range. Nevertheless, the comparison of the Be concentrations between different NW samples is much more precise, due to the same deviation from the exactly defined scattering geometry.

\section{ASSOCIATED CONTENT}

Supporting Information

GaAs NWs produced by 60 mins Be-doped growth and 20 mins undoped growth.

Oxygen distribution map in the NW cross-section

\section{Author Information}

Corresponding Author: Y. Zhang

*E-mail: yunyan.zhang.11@ucl.ac.uk

\section{Author Contributions:}

$\perp$ Z. Sun, A. M. Sanchez, M. Ramsteiner contributed equally to this work.

\section{Notes:}

The authors declare no competing financial interest. 


\section{Acknowledgements:}

The authors acknowledge the support of Leverhulme Trust, National Science Foundation (NSF, DMR-1611341) and Binational Science Foundation (BSF, 2012088)

\section{References:}

(1) Lieber, C. M.; Wang, Z. L. MRS Bull. 2007, 32, 99-108.

(2)Zhang, Y.; Wu, J.; Aagesen, M.; Liu, H. J. Phys. D: Appl. Phys. 2015, 48, 463001.

(3) Yan, R.; Gargas, D.; Yang, P. Nat. Photonics 2009, 3, 569-576.

(4) Dasgupta, N. P.; Sun, J.; Liu, C.; Brittman, S.; Andrews, S. C.; Lim, J.; Gao, H.; Yan, R.; Yang, P. Adv. Mater. 2014, 26, 2137.

(5) Yang, P.; Yan, R.; Fardy, M. Nano Lett. 2010, 10, 1529-1536.

(6)Zheng, G. F.; Patolsky, F.; Cui, Y.; Wang, W. U.; Lieber, C. M. Nat. Biotechnol. 2005, 23, 1294.

(7) Huang, Y.; Duan, X.; Cui, Y.; Lauhon, L. J.; Kim, K. H. \& Lieber, C. M. Science 2001, 294, 1313.

(8) De Franceschi, S.; van Dam, J. A.; Bakkers, E. P. A. M.; Feiner, L. F.; Gurevich, L.; Kouwenhoven, L. P. Appl. Phys. Lett. 2003, 83, 344.

(9) Doh, Y. J.; van Dam, J. A.; Roest, A. L.; Bakkers, E. P. A. M.; Kouwenhoven, L. P.; De Franceschi, S. Science 2005, 309, 272.

(10) Gudiksen, M. S.; Lauhon, L. J.; Wang, J.; Smith, D. C.; Lieber, C. M. Nature 2002, 415, 617.

(11) Holm, J. V.; Jørgensen, H. I.; Krogstrup, P.; Nygård, J.; Liu, H.; Aagesen, M. Nat. Commun. 2013, 4, 1498.

(12) Huang, M. H.; Mao, S.; Feick, H.; Yan, H.; Wu, Y.; Kind, H.; Weber, E.; Russo, R.; Yang, 
P. Science 2001, 292, 1897.

(13) Wu, J.; Ramsay, A.; Sanchez, A. M.; Zhang, Y.; Kim, D.; Brossard, F. S. F.; Salamo, G.

G. Aagesen, M.; Wang, Z.; Liu, H. Nano Lett. 2016, 16, 504.

(14) Wallentin, J.; Borgström, M. T. J. Mater. Res. 2011, 26, 2142.

(15) Wagner, R. S.; Ellis, W. C. Appl. Phys. Lett. 1964, 4, 89-90.

(16) Jacobsson, D.; Panciera, F.; Tersoff, J.; Reuter, M. C.; Lehmann, S.; Hofmann, S.; Dick, K. A.; Ross, F. M. Nature, 2016, 531, 317-322.

(17) Schwarz, K. W.; Tersoff, J.; Kodambaka, S.; \& Ross, F. M. Physical review letters, 2014. $113,055501$.

(18) Connell, J. G.; Yoon, K.; Perea, D. E.; Schwalbach, E. J.; Voorhees, P. W.; Lauhon, L. J. Nano letters, 2012, 13, 199-206.

(19) Amit, I.; Givan, U.; Connell, J. G.; Paul, D. F.; Hammond, J. S.; Lauhon, L. J.; \& Rosenwaks, Y. Nano letters, 2013, 13(6), 2598.

(20) Stichtenoth, D.; Wegener, K.; Gutsche, C.; Regolin, I.; Tegude, F. J.; Prost, W.; Seibt, M.; Ronning, C. Appl. Phys. Lett. 2008, 92, 163107.

(21) Li, H. Y.; Wunnicke, O.; Borgström, M. T.; Immink, W. G. G.; Van Weert, M. H. M.; Verheijen, M. A.; Bakkers, E. P. A. M. Nano lett. 2007, 7, 1144.

(22) Gutsche, C.; Regolin, I.; Blekker, K.; Lysov, A.; Prost, W.; Tegude, F. J. J. Appl. Phys. 2009, 105, 024305.

(23) Zhang, G.; Tateno, K.; Suzuki, S.; Gotoh, H.; Sogawa, T. J. Phys. Chem. C 2011, 115, 2923.

(24) Sager, D.; Gutsche, C.; Prost, W.; Tegude, F. J.; Bacher, G. J. Appl. Phys. 2013, 113, 
174303.

(25) Borgström, M. T.; Norberg, E.; Wickert, P.; Nilsson, H. A.; Trägårdh, J.; Dick, K. A.;

Statkute, G.; Ramvall, P.; Deppert, K.; Samuelson, L. Nanotechnology 2008, 19, 445602.

(26) Suomalainen, S.; Hakkarainen, T. V.; Salminen, T.; Koskinen, R.; Honkanen, M.; Luna,

E.; Guina, M. Appl. Phys. Lett. 2015, 107, 012101.

(27) Casadei, A.; Krogstrup, P.; Heiss, M.; Röhr, J. A.; Colombo, C.; Ruelle, T.; Upadhyay, S.;

Sørensen, C. B.; Nygård, J. \& i Morral, A. F. Appl. Phys. Lett. 2013, 102, 013117.

(28) Wolf, D.; Lichte, H.; Pozzi, G.; Prete, P.; \& Lovergine, N. Applied Physics Letters, 2011, $98,264103$.

(29) Hilse, M.; Ramsteiner, M.; Breuer, S.; Geelhaar, L.; \& Riechert, H. Applied Physics Letters, 2010, 96, 193104.

(30) Chia, A. C. E.; Boulanger, J. P.; \& LaPierre, R. R. Nanotechnology, 2013, 24, 045701.

(31) Morkötter, S.; Jeon, N.; Rudolph, D.; Loitsch, B.; Spirkoska, D.; Hoffmann, E.;

Döblinger, M.; Matich, S.; Finley, J. J.; Lauhon, L. J.; Abstreiter, G.; Koblmüller, G. Nano letters, 2015,.15, 3295.

(32) Ketterer, B.; Mikheev, E.; Uccelli, E.; Fontcuberta i Morral, A. Appl. Phys. Lett. 2010, 97, 223103.

(33) Dubrovskii, V. G.; Xu, T.; Álvarez, A. D.; Plissard, S. R.; Caroff, P.; Glas, F.; Grandidier, B. Nano letters, 2015, 15, 5580.

(34) Ojha, S. K.; Kasanaboina, P. K.; Lewis Reynolds Jr, C.; Rawdanowicz, T. A.; Liu, Y.; White, R. M.; Iyer, S. Journal of Vacuum Science Technology B, Nanotechnology and Microelectronics: Materials, Processing, Measurement, and Phenomena, 2016, 34, 
$02 \mathrm{~L} 114$.

(35) Dufouleur, J.; Colombo, C.; Garma, T.; Ketterer, B.; Uccelli, E.; Nicotra, M. \&

Fontcuberta i Morral, A. Nano lett.; 2010, 10, 1734.

(36) Zhang, Y.; Aagesen, M.; Holm, J. V.; Jørgensen, H. I.; Wu, J.; Liu, H. Nano Lett. 2013, $13,3897-3902$.

(37) Joyce, H. J.; Gao, Q.; Tan, H. H.; Jagadish, C.; Kim, Y.; Zhang, X.; Guo, Y.; Zou, J. Nano lett. 2007, 7, 921.

(38) Kelrich, A.; Sorias, O.; Calahorra, Y.; Kauffmann, Y.; Gladstone, R.; Cohen, S.; Ritter, D. Nano letters, 2016, 16, 2837-2844.

(39) de la Mata, M.; Leturcq, R.; Plissard, S. R.; Rolland, C.; Magén, C.; Arbiol, J.; Caroff, P. Nano letters, 2016, 16, 825-833.

(40) Hocevar, M.; Immink, G.; Verheijen, M.; Akopian, N.; Zwiller, V.; Kouwenhoven, L.; Bakkers, E. arXiv preprint arXiv:1210.8350. 2012

(41) Yuan, X.; Caroff, P.; Wong-Leung, J.; Fu, L.; Tan, H. H.; Jagadish, C. Advanced Materials, 2015, 27, 6096-6103.

(42) Wang, J.; Plissard, S. R.; Verheijen, M. A.; Feiner, L. F.; Cavalli, A.; Bakkers, E. P. Nano letters, 2013, 13, 3802-3806.

(43) Nebol'Sin, V. A.; Shchetinin, A. A. Inorganic materials, 2003, 39, 899-903.

(44) Zhang, Y.; Sanchez, A. M.; Wu, J.; Aagesen, M.; Holm, J. V.; Beanland, R.; Ward, T.; Liu, H. Nano lett. 2015, 15, 3128.

(45) Zheng, C.; Wong-Leung, J.; Gao, Q.; Tan, H. H.; Jagadish, C.; \& Etheridge, J. Nano lett.; 2013, 13, 3742 . 
(46) Jeon, N.; Loitsch, B.; Morkoetter, S.; Abstreiter, G.; Finley, J.; Krenner, H. J.;

Koblmueller, G.; Lauhon, L. J. ACS nano 2015, 9, 8335.

(47)Seidman, D. N.; Stiller, K. MRS bull. 2009, 34, 717.

(48) Sun, Z.; Hazut, O.; Huang, B. C.; Chiu, Y. P.; Chang, C. S.; Yerushalmi, R.; Lauhon, L. J.; Seidman, D. N. Nano lett. 2016, 16, 4490-4500.

(49) Nimmatoori, P., Zhang, Q., Dickey, E. C., \& Redwing, J. M. Nanotechnology, 2008, 20 , 025607.

(50) Boulanger, J. P.; Chia, A. C. E.; Wood, B.; Yazdi, S.; Kasama, T.; Aagesen, M.; LaPierre, R. R. IEEE Journal of Photovoltaics, 2016, 6, 661.

(51) Chen, W.; Pareige, P.; Castro, C.; Xu, T.; Grandidier, B.; Stiévenard, D.; Roca i

Cabarrocas, P. J. Appl. Phys. 2015, 118, 104301.

(52) Komsa, H. P.; Arola, E.; Pakarinen, J.; Peng, C. S.; Rantala, T. T. Phys. Rev. B 2009, 79, 115208.

(53) Wagner, J.; Maier, M.; Murray, R.; Newman, R. C.; Beall, R. B.; Harris, J. J. J. Appl. Phys. 1991, 69, 971.

(54) Murray, R.; Newman, R. C.; Sangster, M. J. L.; Beall, R. B.; Harris, J. J.; Wright, P. J.; Wagner, J.; Ramsteiner, M. J. Appl. Phys. 1989, 66, 2589.

(55) Ihn, S. G.; Song, J. I.; Kim, Y. H.; Lee, J. Y.; Ahn, I. H. IEEE T. Nanotechnol. 2007, 6, 384-389.

(56) Wallentin, J.; Ek, M.; Wallenberg, L. R.; Samuelson, L.; Deppert, K.; Borgstrogm, M. T. Nano Lett. 2010, 10, 4807.

(57) Algra, R. E.; Verheijen, M. A.; Borgström, M. T.; Feiner, L-F.; Immink, G.; van 
Enckevort, W. J. P.; Vlieg, E.; Bakkers, E. P. A. M. Nature 2008, 456, 369.

(58) Wallentin, J.; Mergenthaler, K.; Ek, M.; Wallenberg, L. R.; Samuelson, L.; Deppert, K.;

Pistol, M. E. \& Borgstrom, M. T. Nano lett. 2011, 11, 2286.

(59) Bresse, J. F.; Papadopoulo, A. C. Appl. Phys. Lett. 1987, 51, 183.

(60) Duhamel, N.; Henoc, P.; Alexandre, F.; Rao, E. V. K. Appl. Phys. Lett. 1981, 39, 49.

(61) Enquist, P.; Wicks, G. W.; Eastman, L. F.; Hitzman, C. J. Appl. Phys. 1985, 58, 4130.

(62) Ivanov, S. V.; Kop'ev, P. S.; Ledentsov, N. N. J. Cryst. growth 1991, 108, 661.

(63) Morita, M.; Kobayashi, K.; Suzuki, T.; Okano, Y. Japanese J. Appl. Phys. 1989, 28, 553.

(64) Zhang, Y.; Sanchez, A. M.; Sun, Y.; Wu, J.; Aagesen, M.; Huo, S.; Liu, H. Nano lett.; 2016, 16, 1237.

(65) Zhang, Y.; Wu, J.; Aagesen, M.; Holm, J.; Hatch, S.; Tang, M.; Huo, S.; Liu, H. Nano lett. 2014, 14, 4542 .

(66) Sun, Z.; Tzaguy, A.; Hazut, O.; Lauhon, L. J.; Yerushalmi, R.; Seidman, D. N. Nano Lett. 2017, DOI: DOI: 10.1021/acs.nanolett.7b03391 